\title{
Black Dipole or White Dipole: Using Susceptibility Phase Imaging to Differentiate Cerebral Microbleeds from Intracranial Calcifications
}

\author{
(D) C.-L. Weng, (D)Y. Jeng, (DY.-T. Li, (D)C.-J. Chen, and (DD.Y.-T. Chen
}

\begin{abstract}
BACKGROUND AND PURPOSE: Phase imaging helps determine a lesion's susceptibility. However, various inhomogenous phase patterns could be observed in the serial phase images of a lesion and render image interpretation challenging. We evaluated the diagnostic accuracy of differentiating cerebral microbleeds and calcifications from phase patterns in axial locations.
\end{abstract}

\begin{abstract}
MATERIALS AND METHODS: This study retrospectively enrolled 31 consecutive patients undergoing both CT and MR imaging for acute infarction exhibiting dark spots in gradient-echo magnitude images. Six patients had additional quantitative susceptibility mapping images. To determine their susceptibility, 2 radiologists separately investigated the phase patterns in the border and central sections and quantitative susceptibility mapping of dark spots. Sensitivity and specificity were compared using the McNemar test. Interobserver reliability and correlation analysis were determined using the $\kappa$ coefficient and Pearson correlation coefficient, respectively.

RESULTS: Among 190 gradient-echo dark spots, 62 calcifications and 128 cerebral microbleeds were detected from CT. Interobserver reliability was higher for the border phase patterns $(\kappa=1)$ than for the central phase patterns $(\kappa=0.77, P<.05)$. The sensitivity and specificity of the border phase patterns in identifying calcifications were higher than those of the central phase patterns $(98.4 \%$ and $100 \%$ versus $79 \%$ and $83.6 \%$ ), particularly for lesions $>2.5 \mathrm{~mm}$ in diameter (100\% and $100 \%$ versus $66.7 \%$ and $61.1 \%$ ). The same values were obtained using quantitative susceptibility mapping for identification (100\% and $100 \%)$. A high correlation between the size and susceptibility of cerebral microbleeds and calcifications suggested that greater phase changes may be caused by larger lesions.
\end{abstract}

CONCLUSIONS: The border phase patterns were more accurate than the central phase patterns in differentiating calcifications and cerebral microbleeds and was as accurate as quantitative susceptibility mapping.

ABBREVIATIONS: CMB = cerebral microbleed; GRE = gradient recalled-echo; QSM = quantitative susceptibility mapping

erebral microbleed $(\mathrm{CMB})$ is common in patients with cerebrovascular diseases, traumatic brain injury, and Alzheimer disease and in the healthy elderly population. ${ }^{1}$ Identifying CMBs is helpful for etiologic diagnosis, treatment optimization, and prognosis prediction. ${ }^{2,3}$ For example, CMBs in the deep nuclei are more often related to hypertensive arteriopathy than to cerebral amyloid angiopathy, and the number of baseline and recurrent $\mathrm{CMBs}$ is associated with future intracerebral hemorrhage and

Received February 17, 2020; accepted after revision May 1.

From the Department of Radiology (C.-L.W., Y.-T.L., C.-J.C., D.Y.-T.C.), Shuang Ho Hospital, Taipei Medical University, New Taipei City, Taiwan; Department of Medical Imaging (Y.J.), National Taiwan University Hospital, Taipei, Taiwan; Department of Medical Imaging (Y.J.), National Taiwan University Hospital HsinChu Branch, Hsin Chu City, Taiwan; Department of Radiology (C.-J.C., D.Y.-T.C.), School of Medicine, Translational Imaging Research Center (Y.-T.L.), and College of Medicine, Neuroscience Research Center (Y.-T.L.), Taipei Medical University, Taipei, Taiwan

Please address correspondence to David Yen-Ting Chen, MD, Department of Radiology, Shuang Ho Hospital, Taipei Medical University, No. 291, Zhongzheng Rd, Zhonghe District, New Taipei City, 23561 Taiwan; e-mail: b91401019@ntu.edu.tw

http://dx.doi.org/10.3174/ajnr.A6636 ischemic stroke in patients with small-vessel disease or the possibility of hemorrhagic transformation for patients with stroke before thrombolytic treatment. ${ }^{4-6} \mathrm{CMBs}$ can be accurately detected through $\mathrm{T}^{\star}$-weighted gradient recalled-echo (GRE) imaging, CT, and conventional T2-weighted imaging. ${ }^{7}$ However, CMBs can appear nearly identical to calcifications in GRE magnitude images with their dark and rounded appearance, which may cause diagnostic confusion during image interpretation. Furthermore, calcifications typically have nonspecific signals in other conventional MR images, such as T1 and T2 spin-echo imaging. Additional CT may be necessary for a definitive diagnosis of calcification despite the risk of exposing patients to additional ionizing radiation.

CMBs and calcifications can be distinguished using GRE phase imaging because it reflects the regional magnetic field perturbations caused by lesions with different susceptibilities. ${ }^{7-11}$ Paramagnetic CMBs appear dark, whereas diamagnetic calcifications appear bright on phase images in the right-handed system, which is used by vendors such as GE Healthcare and Philips Healthcare. ${ }^{8-11}$ By contrast, $\mathrm{CMBs}$ appear bright and calcifications appear dark on 


\section{Microbleed or Calcification?}

A GRE Magnitude Image

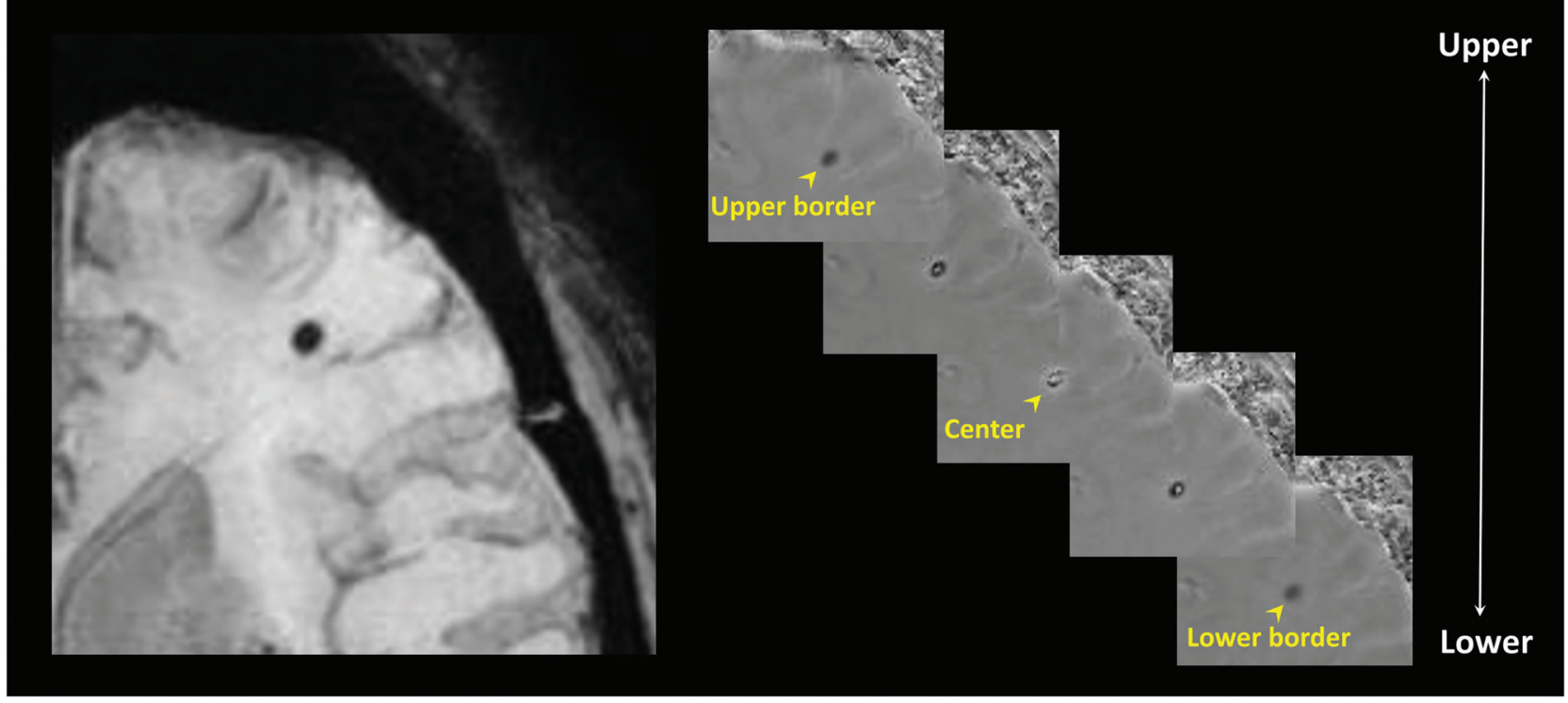

FIG 1. Microbleed or calcification? A, GRE magnitude image shows a dark spot in the left frontal white matter. $B$, GRE phase images of the corresponding lesion reveal various phase patterns from the cranial-to-caudal direction. These appear totally dark at the upper and lower border sections; however, a mixed dark and bright pattern is visible in the center section. The heterogeneous phase pattern hinders interpretation of whether the lesion is a microbleed or calcification.

phase images in the left-handed system, which is used by vendors such as Siemens and Canon. Nevertheless, in daily practice, observing inhomogeneous patterns in 1 phase image or different patterns in serial phase images for a single lesion renders image interpretation challenging (Fig 1). Characterizing the susceptibility of a lesion using 1 section of a phase image is difficult. ${ }^{8}$ This challenge can be overcome by calculating the local susceptibility change caused by a lesion through quantitative susceptibility mapping (QSM), ${ }^{8,12}$ which was reported to outperform GRE phase imaging for differentiating intracranial calcifications and hemorrhages. ${ }^{8}$ However, QSM involves a complicated unwrapping and deconvolution process that is time-consuming. In addition, QSM remains unstandardized for routine clinical use. ${ }^{13-17}$ By contrast, GRE phase images can be readily generated during routine image acquisition on clinical MR scanners.

In this study, we determined the diagnostic accuracy and susceptibility properties of dark spots in GRE magnitude images using GRE phase images at the border and central slices of the lesion. We studied the relationship between the size and susceptibility of a GRE dark spot and explain serial changes in the axial phase patterns of lesions.

\section{MATERIALS AND METHODS \\ Patient Selection}

This retrospective study was approved by our institutional review board Taipei Medical University-Joint Institutional Review Board. From March 2015 to January 2017, we enrolled 36 consecutive patients undergoing both brain $\mathrm{CT}$ and $\mathrm{MR}$ imaging within
3 days of experiencing acute infarction and exhibiting multiple dark spots in GRE magnitude images. Among these patients, 6 had additional QSM images. We excluded 5 patients because of poor GRE image quality. Thus, a total of $31 \mathrm{CT}$ and $31 \mathrm{MR}$ images were retrospectively reviewed. The mean age of the 31 patients (male patients, $n=13$ ) was $69 \pm 10.4$ years (range, 47-89 years). The mean interval between CT and MR imaging was $38.9 \pm 15.4$ hours (range, $8-71$ hours).

\section{Image Acquisition and Postprocessing}

MR imaging was performed on a 3T scanner (Discovery MR750; GE Healthcare) with an 8-channel head coil. GRE images were acquired using susceptibility-weighted angiography and a 3D flow-compensated multiecho GRE sequence with the following parameters: TR, $37.8 \mathrm{~ms}$; TE, $25 \mathrm{~ms}$, with 6 echoes; FOV, $230 \mathrm{~mm}^{2} ; 512 \times 512$ matrix; flip angle, $15^{\circ}$; and section thickness, $2.5 \mathrm{~mm}$. CT was performed on a 16-detector CT scanner (BrightSpeed VCT; GE Healthcare) with the following parameters: $310 \mathrm{mAs}, 120 \mathrm{kV}, 5-\mathrm{mm}$ section thickness, and $512 \times 512$ matrix. All images were acquired in the axial plane parallel to the orbitomeatal line. GRE phase images were automatically generated using high-pass filtering to remove background phase variation from the source images in the MR imaging scanner. ${ }^{18}$ Coronal reformation of the GRE phase images was performed on a standard workstation (AW Workstation; GE Healthcare). QSM was generated from the same GRE images using the iterative least-squares regression method with STI-Suite (Version 2.1) (https://people.eecs.berkeley.edu/ ${ }^{\sim}$ chunlei.liu/software.html) on a personal computer. ${ }^{13}$ 


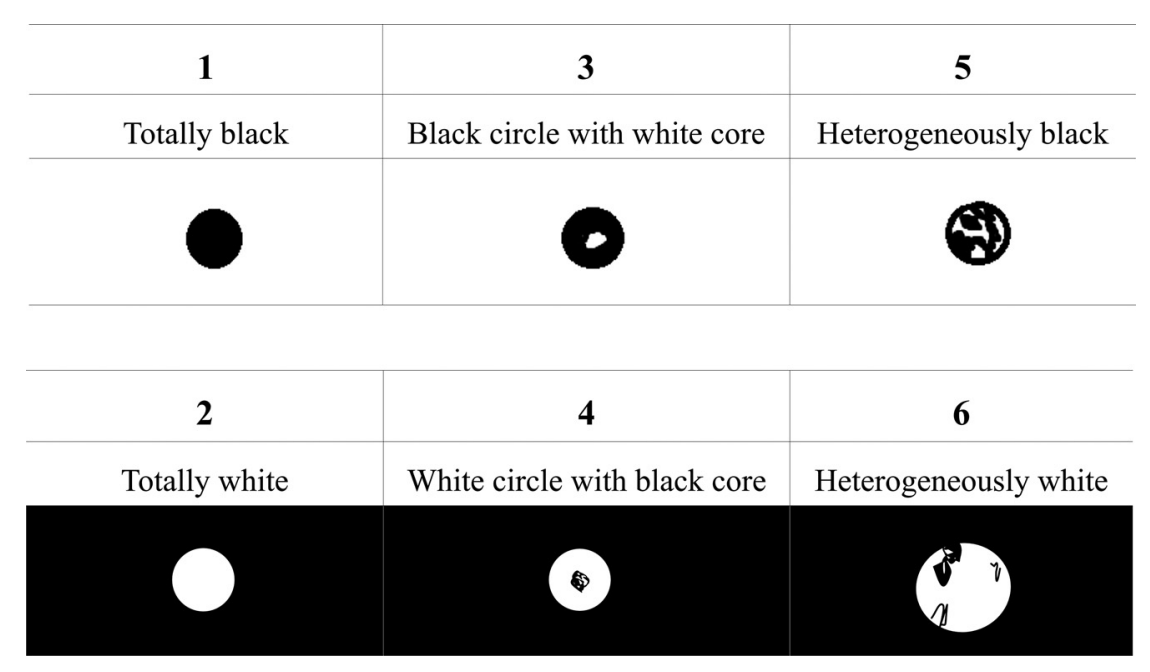

FIG 2. Axial phase patterns of GRE dark spots. The 6 axial phase patterns include the following: pattern 1, totally black; pattern 2, totally white; pattern 3, black circle with white core; pattern 4 , white circle with black core; pattern 5 , heterogeneously black; and pattern 6 , heterogeneously white. Lesions with phase patterns 1, 3, and 5 were interpreted as paramagnetic microbleeds in which $>50 \%$ of the area is dark. Lesions with phase patterns 2,4 , and 6 were interpreted as diamagnetic calcifications in which $>50 \%$ of the area appeared bright.

\section{Reference Standards}

A radiologist (with 3 years of experience) reviewed all the GRE magnitude images to detect targeted lesions with the following characteristics: 1) dark, 2) round or oval in the brain, and 3) $<10 \mathrm{~mm}$ in diameter. GRE dark spots in the globus pallidus were excluded to prevent a susceptibility contribution from iron deposition. ${ }^{8,9}$ The size and anatomic location of each lesion were documented. CT was used as the criterion standard for identifying the presence or absence of calcifications. Calcifications are lesions with a density of at least $100 \mathrm{HU}$ in CT images, and CMBs are invisible on CT images. In patients with QSM images, the susceptibility value of each GRE dark spot was recorded.

\section{Phase Pattern and Interpretation}

Subsequently, 2 other radiologists (reader 1 with 6 years of experience and reader 2 with 8 years of experience) who were unaware of each other's interpretation of the nature of the lesion independently categorized the phase pattern of each lesion at the central and upper/lower border slices, as illustrated in Fig 2. The central and border phase patterns indicated the signal intensity of the axial section at the center and edge of each dark spot on phase images, respectively. From our observations, the upper and lower border slices always appeared in the same pattern; therefore, we only recorded the upper border pattern. Lesions categorized into phase patterns 1 (total black), 3 (black circle with white core), or 5 (heterogeneous black) were interpreted as paramagnetic CMBs because the dominant signal was dark ( $>50 \%)$. Lesions categorized into phase pattern 2 (total white), 4 (white circle with black core), or 6 (heterogeneous white) were interpreted as diamagnetic calcifications according to the opposite dominant signal. For lesions on QSM, the 2 readers independently identified the susceptibility property of each lesion. Paramagnetic lesions were bright, whereas diamagnetic lesions were dark. The 2 readers reached a consensus reading of the central phase pattern, border phase pattern, and QSM of each lesion.

\section{Statistical Analyses}

We assessed interobserver reliability using the $\kappa$ statistic to evaluate the consistency of the 2 radiologists' readings of border and central phase patterns. The $Z$-test was used to evaluate the difference in $\kappa$ statistics between the 2 phase patterns. The sensitivity and specificity of border and central phase patterns for identifying calcifications were computed. For lesions on QSM, we also calculated the sensitivity and specificity of QSM in identifying calcifications, which were then compared with those of the phase images. The McNemar test was used to evaluate differences in sensitivity and specificity to identify calcifications among the border phase pattern, central phase pattern, and QSM. Furthermore, we evaluated the relationship between the size and susceptibility of a lesion using the Pearson correlation coefficient. Statistical analyses were performed using SPSS for Windows (Version 16.0; IBM). A value of $P<.05$ indicated a statistically significant difference.

\section{RESULTS}

A total of 190 dark spots were detected in GRE magnitude images, which comprised 62 calcifications and $128 \mathrm{CMBs}$ from the CT. All CMBs were detected in the brain parenchyma and had a mean diameter of $2.6 \pm 1.0 \mathrm{~mm}$ (range, $1.5-5.8 \mathrm{~mm}$ ). The numbers of calcifications observed were as follows: 10 in the brain parenchyma, 38 in the pineal gland, 7 in the choroid plexus, 6 in the cerebral falx, and 1 on the arterial wall. The mean size was $3.2 \pm 1.4 \mathrm{~mm}$ (range, $1.5-6.9 \mathrm{~mm}$ ).

Table 1 summarizes the central and border phase patterns of CMBs and calcifications. A consensus was reached that in the 128 CMBs, all border phase images revealed paramagnetic phase pattern 1 . Of the central phase images, $107(83.6 \%)$ had paramagnetic phase patterns, and 21 (16.4\%) had diamagnetic phase patterns. For the 62 calcifications, 61 (98.4\%) of the border phase images had diamagnetic phase pattern 2, and only 1 (1.6\%) had paramagnetic phase pattern 1 . Of the central phase images, 49 (79.1\%) had diamagnetic phase patterns, and 13 (20.9\%) had paramagnetic phase patterns. Overall, the border phase images had only homogeneous phase patterns (ie, patterns 1 and 2), whereas central phase images were more variable and exhibited more heterogeneous phase patterns (ie, patterns 3-6). Accordingly, interobserver variability in interpreting the phase pattern was higher for border phase patterns $(\kappa=1)$ than for central phase patterns $(\kappa=0.77 ; P<.05)$. Furthermore, the heterogeneity and variability of the central phase patterns depended on the size of the lesion. In lesions $<2.5 \mathrm{~mm}$ in diameter, 99 (99\%) of the 100 lesions exhibited homogeneous phase patterns, and only 1 had 
Table 1: Phase patterns of cerebral microbleeds and intracranial calcifications at the border and central slices

\begin{tabular}{|c|c|c|c|c|c|c|}
\hline \multirow[b]{2}{*}{ Phase Pattern } & \multicolumn{3}{|c|}{ Microbleeds $(n=128)$} & \multicolumn{3}{|c|}{ Calcifications ( $n=62)$} \\
\hline & All sizes & $<2.5 \mathrm{~mm}$ & $\geq 2.5 \mathrm{~mm}$ & All sizes & $<2.5 \mathrm{~mm}$ & $\geq 2.5 \mathrm{~mm}$ \\
\hline \multicolumn{7}{|l|}{ Border phase pattern } \\
\hline 1) Totally black & 128 & 74 & 54 & 1 & 1 & 0 \\
\hline 2) Totally white & 0 & 0 & 0 & 61 & 25 & 36 \\
\hline 3) Black circle with white core & 0 & 0 & 0 & 0 & 0 & 0 \\
\hline 4) White circle with black core & 0 & 0 & 0 & 0 & 0 & 0 \\
\hline 5) Heterogeneously black & 0 & 0 & 0 & 0 & 0 & 0 \\
\hline 6) Heterogeneously white & 0 & 0 & 0 & 0 & 0 & 0 \\
\hline \multicolumn{7}{|l|}{ Central phase pattern } \\
\hline 1) Totally black & 80 & 73 & 7 & 2 & 1 & 1 \\
\hline 2) Totally white & 0 & 0 & 0 & 27 & 25 & 2 \\
\hline 3) Black circle with white core & 10 & 0 & 10 & 0 & 0 & 0 \\
\hline 4) White circle with black core & 3 & 0 & 3 & 9 & 0 & 9 \\
\hline 5) Heterogeneously black & 17 & 1 & 16 & 11 & 0 & 11 \\
\hline 6) Heterogeneously white & 18 & 0 & 18 & 13 & 0 & 13 \\
\hline
\end{tabular}

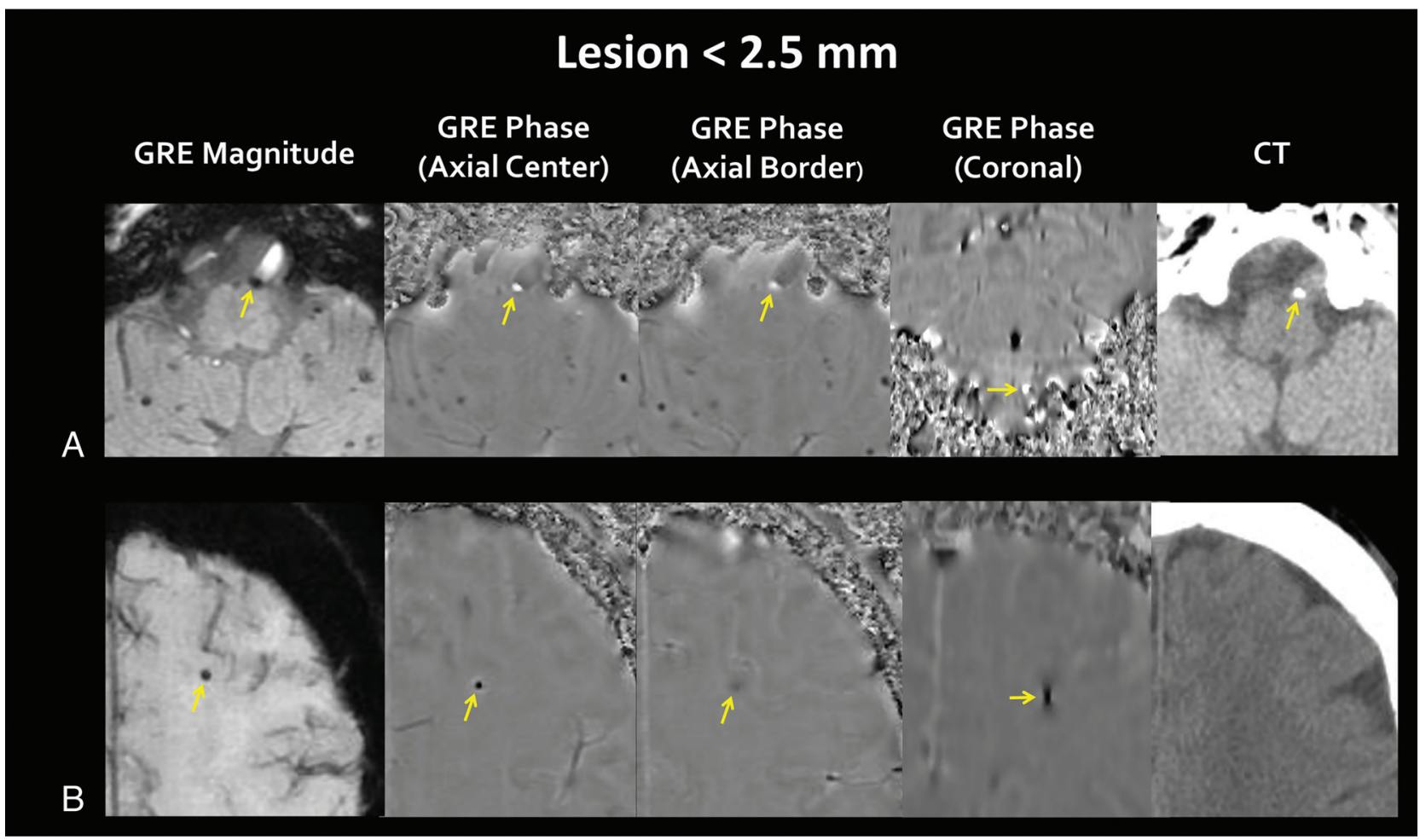

FIG 3. Phase patterns of calcifications and microbleeds $<2.5 \mathrm{~mm}$ in diameter. $A$, A small dark spot (arrow) attached to the left distal vertebral artery is shown in the GRE magnitude image. It is totally white at the central with border slices in the axial phase image, which suggest a diamagnetic lesion. CT confirms this as arterial wall calcification. B, A small dark spot (arrow) located at the left frontal subcortical white matter in the GRE magnitude image. It is totally dark at the central with border slices of the axial phase image, which suggest a paramagnetic lesion. Coronal phase imaging reveals a black dipole with a pair of faint bright side wings. The bright side wings are the faint, peripheral, bright rim around the dark lesion in the axial central phase image. CT reveals no corresponding lesion and confirms it to be a microbleed.

heterogeneous phase patterns in the central phase images. However, in lesions at least $2.5 \mathrm{~mm}$ in diameter, 80 (88.9\%) of 90 lesions had heterogeneous phase patterns, and 10 (11.1\%) had homogeneous phase patterns. Figure 3 illustrates examples of a $\mathrm{CMB}$ and calcification $<2.5 \mathrm{~mm}$, and Fig 4 presents examples of lesions of $>2.5 \mathrm{~mm}$.

Table 2 summarizes the sensitivity and specificity of the border and central phase patterns used to identify calcifications. The border phase pattern had a sensitivity of $98.4 \%$ and a specificity of $100 \%$, which were higher than those of the central phase patterns (sensitivity $=79 \%, P=.002$; specificity $=83.6 \%, P=<.001$ ). Diagnostic accuracy depended on the size of the lesion. For lesions at least $2.5 \mathrm{~mm}$ in diameter, the sensitivity and specificity of the central phase pattern decreased to $66.7 \%$ and $61.1 \%$, respectively, which were lower than those of the border phase pattern (sensitivity $=100 \%$, $P=.002$; specificity $=100 \%, P=<.001)$. However, for lesions $<2.5 \mathrm{~mm}$ in diameter, both sensitivity and specificity were very high for the central phase pattern $(96.2 \%$ and $100 \%$, respectively) and the border phase pattern (96.2\% and $100 \%$, 


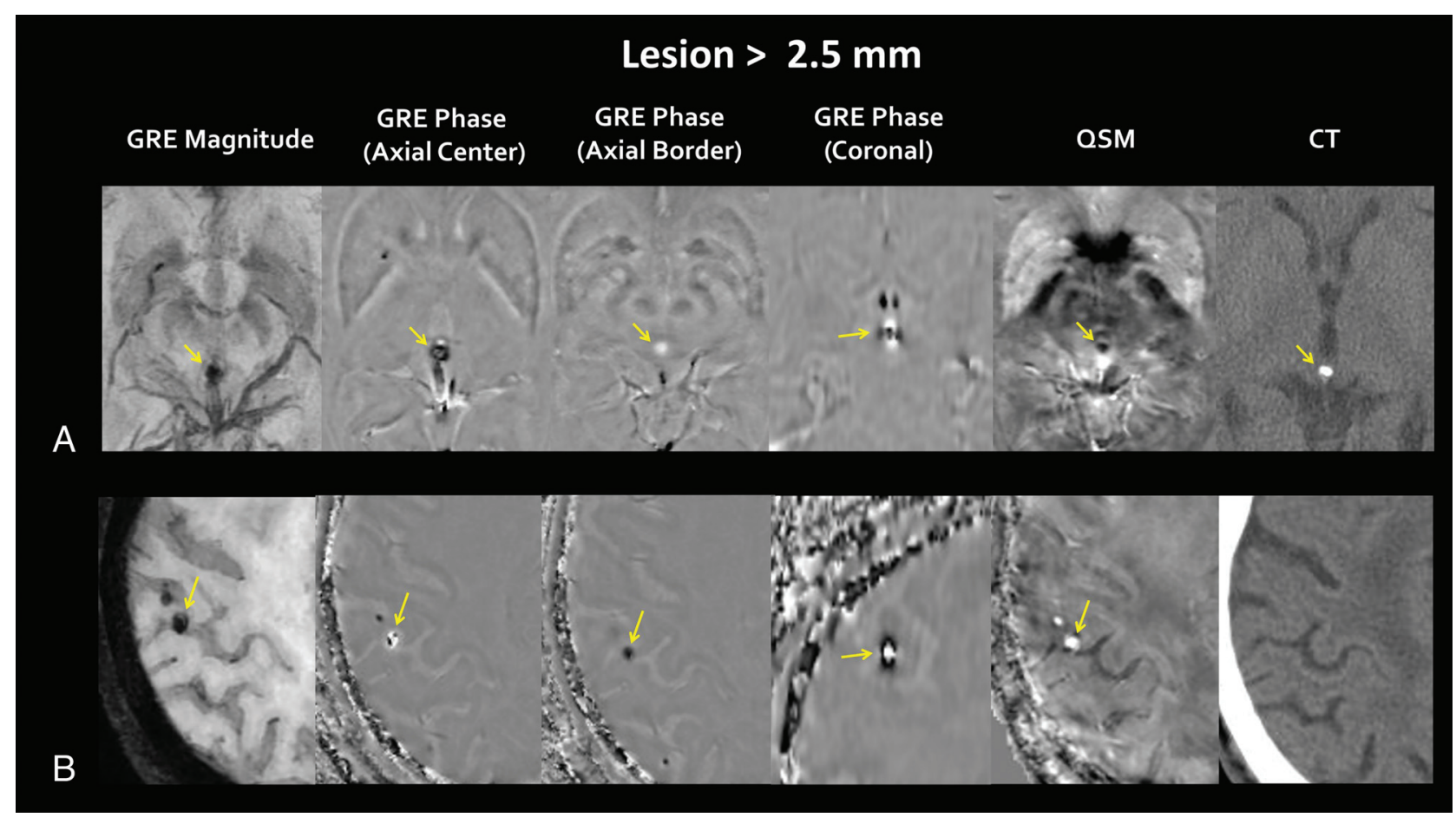

FIG 4. Phase patterns of calcifications and microbleeds of $>2.5 \mathrm{~mm}$ in diameter. $A$, A dark nodule (arrow) in the pineal region is displayed in the GRE magnitude image. It is heterogeneously black in the axial central phase image, whereas it is totally white in the axial border phase image. The inconsistent finding of the axial phase pattern hinders differentiation between a calcification and microbleed. Coronal phase imaging displays a white dipole more clearly, with a black core and side wings (arrow). QSM indicates a dark spot (arrow) at the corresponding site, which indicates a diamagnetic lesion. CT confirms it to be a pineal calcification. B, A dark nodule (arrow) at the right temporal subcortical white matter is presented in the GRE magnitude image. It is heterogeneously white in the axial central phase image, whereas it is totally black in the axial border phase images. The inconsistent finding hinders differentiation between a calcification and microbleed. Coronal phase imaging reveals a black dipole with a white core and side wings (arrow), and QSM indicates a bright spot (arrow) at the corresponding site, which suggests a paramagnetic lesion. CT confirms this to be a microbleed.

Table 2: Diagnostic accuracy of the border and central phase patterns and QSM for identifying calcification

\begin{tabular}{|c|c|c|c|c|c|c|c|c|c|c|c|c|}
\hline \multirow[b]{3}{*}{ Lesion Diameter } & \multicolumn{6}{|c|}{ All Lesions $(n=190)$} & \multicolumn{6}{|c|}{ Lesions with QSM ( $n=46)$} \\
\hline & \multicolumn{2}{|c|}{ Border Phase } & \multicolumn{2}{|c|}{ Central Phase } & \multicolumn{2}{|c|}{$P$ Value } & \multicolumn{2}{|c|}{ Border Phase } & \multicolumn{2}{|c|}{ QSM } & \multicolumn{2}{|c|}{$P$ Value } \\
\hline & $\begin{array}{l}\text { SEN } \\
(\%)\end{array}$ & $\begin{array}{l}\text { SPE } \\
(\%)\end{array}$ & $\begin{array}{l}\text { SEN } \\
\text { (\%) }\end{array}$ & $\begin{array}{l}\text { SPE } \\
(\%)\end{array}$ & SEN & SPE & $\begin{array}{l}\text { SEN } \\
\text { (\%) }\end{array}$ & $\begin{array}{l}\text { SPE } \\
\text { (\%) }\end{array}$ & $\begin{array}{l}\text { SEN } \\
\text { (\%) }\end{array}$ & $\begin{array}{l}\text { SPE } \\
\text { (\%) }\end{array}$ & SEN & SPE \\
\hline All sizes $(n=190)$ & 98.4 & 100 & 79.0 & 83.6 & .002 & $<.001$ & 100 & 100 & 100 & 100 & 1 & 1 \\
\hline$<2.5 \mathrm{~mm}(n=100)$ & 96.2 & 100 & 96.2 & 100 & 1 & 1 & 100 & 100 & 100 & 100 & 1 & 1 \\
\hline$\geq 2.5 \mathrm{~mm}(n=90)$ & 100 & 100 & 66.7 & 61.1 & .002 & $<.001$ & 100 & 100 & 100 & 100 & 1 & 1 \\
\hline
\end{tabular}

Note:-SEN indicates sensitivity; SPE, specificity; QSM, quantitative susceptibility mapping.

respectively). For 46 lesions appearing through QSM, the sensitivity and specificity of the border phase pattern (100\% and $100 \%$, respectively) were the same as those appearing in QSM (100\% and $100 \%$, respectively).

In quantitative analysis, the mean susceptibility of CMBs and calcifications was $0.23 \pm 0.17 \mathrm{ppm}$ (range, $0.01-0.61 \mathrm{ppm}$ ) and $-0.24 \pm 0.1 \mathrm{ppm}$ (range, $-0.10-0.41 \mathrm{ppm}$ ), respectively. A high positive correlation was observed between the diameter and susceptibility values of lesions in CMBs $(r=0.721 ; P<$ .001 ; Fig $5 A$ ), and a strong negative correlation was observed between the diameter and susceptibility values of lesions in calcifications $(r=-0.824 ; P=.003$; Fig $5 B)$. These results suggested that larger $\mathrm{CMBs}$ and calcifications have higher susceptibility, which may cause more local phase shifts than smaller CMBs and calcifications.

\section{DISCUSSION}

Our study indicated that the border phase patterns had higher interobserver reliability than the central phase patterns. The border phase patterns were homogeneous, whereas the central phase patterns were relatively heterogeneous and variable. The border phase patterns had higher diagnostic accuracy in differentiating calcifications and CMBs than the central phase patterns, particularly for lesions of $>2.5 \mathrm{~mm}$ in diameter. Moreover, the border phase patterns had the same diagnostic accuracy as QSM in differentiating calcifications and CMBs.

The phase pattern of a GRE dark spot is determined by its susceptibility. The local magnetic field change $(\Delta B)$, caused by a spheric paramagnetic/diamagnetic lesion at an arbitrary point $(r)$ from the center of the lesion, is described in Equation 1 for within the lesion and Equation 2 for outside the lesion: ${ }^{19}$

AJNR Am J Neuroradiol 41:1405-13 Aug 2020 www.ajnr.org 

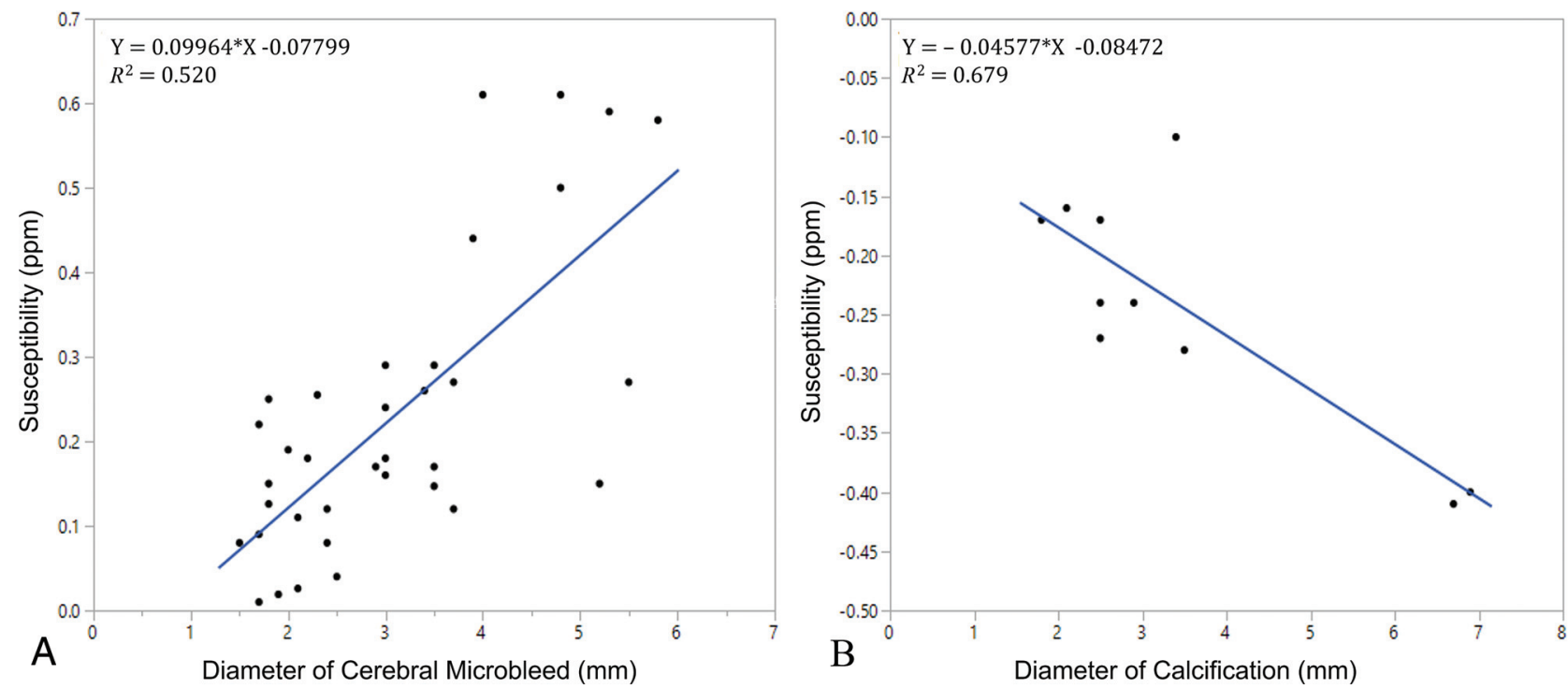

FIG 5. Correlation between the diameter and susceptibility of lesions. A, A strong positive correlation is observed between the diameter and susceptibility of lesions in $\mathrm{CMBs}(r=0.72, P<.001)$. B, A strong negative correlation is observed between the diameter and susceptibility of lesions in calcifications $(r=-0.824, P=.003)$.

Lesion $<2.5 \mathrm{~mm}$

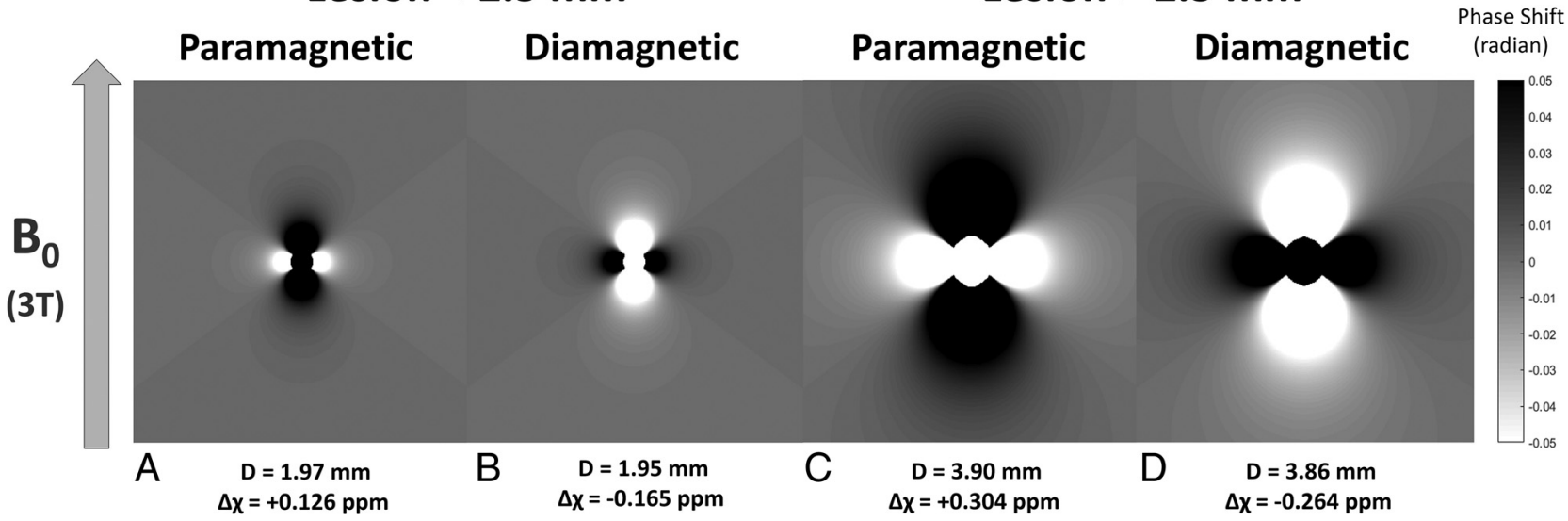

FIG 6. $2 \mathrm{D}$ simulation of the local phase shift around lesions smaller and larger than $2.5 \mathrm{~mm}$ in diameter. The simulation was based on Equations 1 and 2 . The phase shift is represented in the radians, and phase aliasing occurs when the phase shift exceeds $+\pi$ or $-\pi$ radians. The parameters are the same as those in our clinical setting: $B_{0}, 3 T ; T E, 25 \mathrm{~ms}$; and gyromagnetic ratio, $42.58 \times 10^{6} \mathrm{~Hz} / \mathrm{T}$. The relative susceptibility $(\Delta \chi)$ and diameter (D) of the lesion are the mean values of the lesions in each group in our study. In lesions $<2.5 \mathrm{~mm}$ in diameter, the simulated phase image reveals a black dipole with white side wings in the paramagnetic lesion $(A)$ and a white dipole with black side wings in the diamagnetic lesion $(B)$. In lesions $>2.5 \mathrm{~mm}$ in diameter, the relative susceptibility is higher and the diameter is larger, which causes a larger phase shift that exceeds the aliasing threshold within the lesion. This results in a black dipole with a white core from phase aliasing, the white side wings in the paramagnetic lesion (C), and a white dipole with a black core and black side wings in the diamagnetic lesion (D).

1)

$$
\Delta B(r)=\frac{\Delta \chi \times B_{0}}{3} \times 2, \text { for } r \leq \frac{D}{2}
$$

2)

$\Delta B(r)=\frac{\Delta \chi \times B_{0}}{3} \times\left(\frac{D}{2 r}\right) \times(3 \cos 2 \theta-1)$, for $r>D / 2$,

where $\Delta \chi$ is the relative susceptibility of the lesion compared with water, $D$ is the diameter of the lesion, $B_{0}$ is the main magnetic field, and $\theta$ is the angle between $r$ and $\mathrm{B}_{0}$. Furthermore, the local phase shift caused by a lesion in GRE phase images is proportional to the local magnetic field change multiplied by the gyromagnetic ratio then multiplied by the $\mathrm{TE} .{ }^{8}$ In the $2 \mathrm{D}$ phase imaging simulation, a paramagnetic lesion induced an increased local phase shift parallel to the main magnetic field on the superior and inferior sides and antiparallel on the lateral side (Fig 6A), whereas a diamagnetic lesion caused the opposite change (Fig $6 B) .{ }^{8}$ Because the direction of the main magnetic field in clinical MR imaging examinations is parallel to the body axis, the coronal phase image more favorably represented the susceptibility effect of a lesion than the axial phase image. A paramagnetic $\mathrm{CMB}$ 

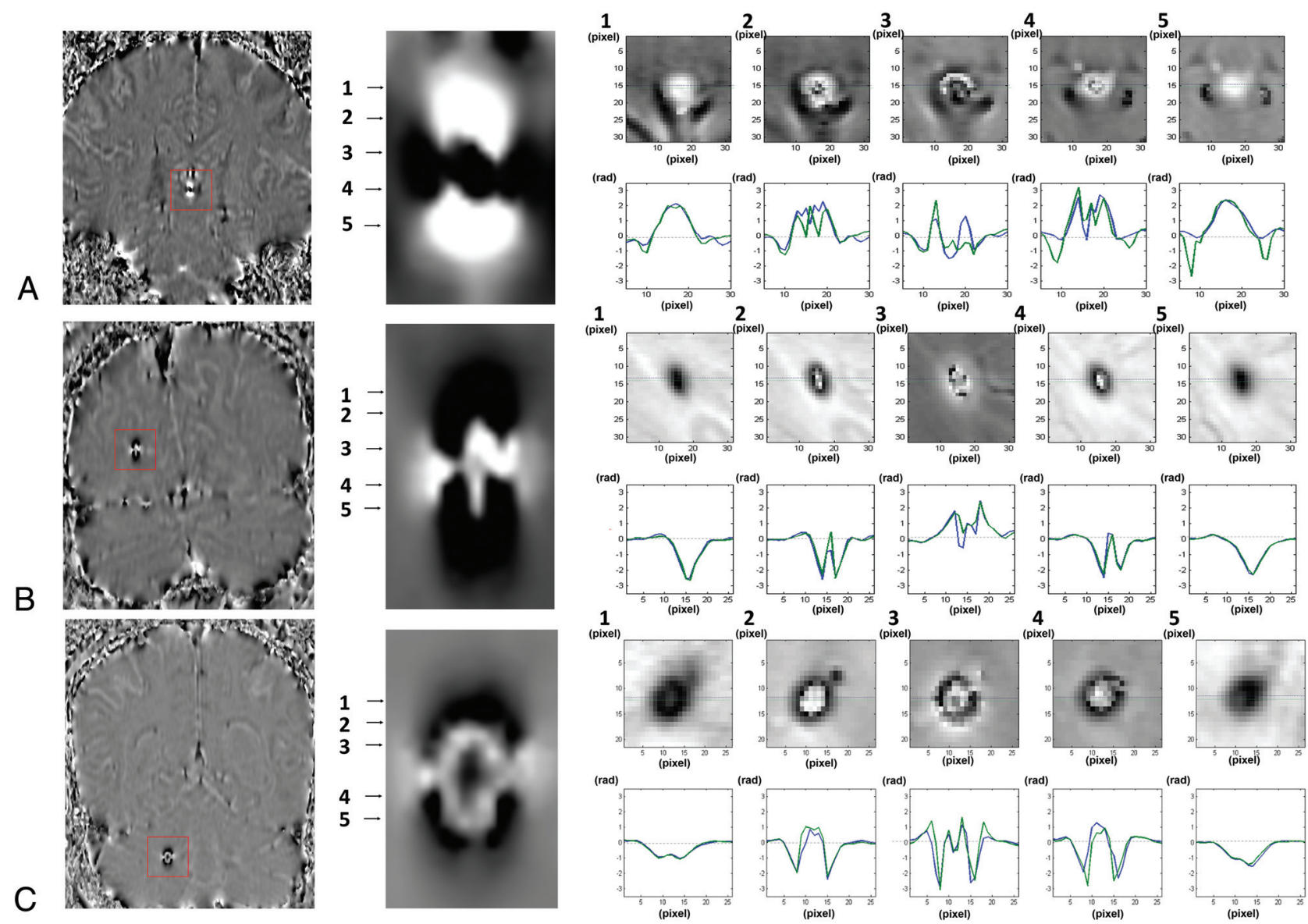

FIG 7. Serial changes in the axial phase pattern in paramagnetic and diamagnetic dipoles. A, A diamagnetic dipole, which represents a pineal calcification, is displayed in the coronal phase image (left) and magnified image (middle). The white dipole with a black core and side wings in the coronal phase image explains serial changes in the axial phase patterns (right upper images) in the corresponding 5 levels: 1, totally white; 2, white circle with black core; 3 , heterogeneously black (epicenter); 4 , white circle with black core; and 5 , totally white (lower border). The phase shift diagram (right lower images) crossing the center of the lesion reveals that when the positive phase shift is large enough to exceed $\pi$ radians, it aliases toward $-\pi$ radians and appears to be partially dark in the phase images near the central slices (2, 3, and 4 of the right lower images). $B$, A paramagnetic black dipole, which represents a microbleed in the white matter of the right cerebral hemisphere, presents the opposite pattern in the coronal and axial phase images and phase shift diagrams. C, A paramagnetic black dipole larger than that in $B$ shows an alternating "black in white" core in the coronal phase (middle) and axial phase images (2, 3, and 4 of the right upper images). The phase shift diagram indicates that when the negative phase shift is large enough to exceed $-\pi$ radians, it aliases toward $+\pi$ radians ( 2 and 4 of the right lower images), and if the phase shift exceeds $+\pi$ radians after the first aliasing, it aliases backward by $-\pi$ radians ( 3 of the right lower images), which causes the alternating black in white core pattern in the center of the black dipole.

appeared as a black dipole with a pair of bright wings, whereas a diamagnetic calcification appeared as a white dipole with black wings.

The strong correlation between the size and susceptibility of CMBs and calcifications suggested that large lesions cause high phase shifts. If the positive phase shift caused by a large paramagnetic lesion exceeds $\pi$ radians, it aliases to $-\pi$ radians and appears to be bright rather than dark in the phase image. ${ }^{20}$ Phase aliasing is most likely to occur in the center of a lesion because the local phase shift is most severe inside lesions and decreases with the cube of the distance from the center of the lesion to outside the lesion (Equations 1 and 2). Therefore, a small white core caused by phase aliasing could be observed in the center of the black dipole in the $2 \mathrm{D}$ phase simulation of a large paramagnetic lesion with high susceptibility (Fig 6C). Notably, we observed the same pattern in the coronal reformation of the phase images in paramagnetic CMBs of $>2.5 \mathrm{~mm}$ in diameter (Fig 7B). A small black core caused by phase aliasing was observed in the center of a white dipole in the $2 \mathrm{D}$ phase simulation of a large diamagnetic lesion with high susceptibility (Fig 6D) and in our clinical coronal phase images when the calcifications were $>2.5 \mathrm{~mm}$ (Fig $7 A$ ). Furthermore, when a lesion had even higher susceptibility, the local phase shift could be large enough to alias twice, which resulted in an alternating white and black core and led to a more heterogeneous phase pattern in the central section (Fig 7C). Other factors may have also led to heterogeneity in the phase pattern, including the noise of $\mathrm{T} 2{ }^{*}$-weighted GRE imaging, high-pass filtering (particularly for large lesions), and the heterogeneous density of calcification and hemosiderin deposition in CMBs. ${ }^{8,10,11,21}$ Therefore, in this study, central phase patterns were more heterogeneous and variable, particularly for lesions of $>2.5 \mathrm{~mm}$ in diameter, and were unreliable in determining the susceptibility of the lesions. 
The border phase patterns achieved the same sensitivity and specificity for identifying calcifications as QSM. The border section was far from the center of the lesion and, by definition, presented the smallest perceptible phase shift. Therefore, the border phase patterns were not affected by aliasing and were always homogeneous and accurate in reflecting the susceptibility of the lesions. Chen et $\mathrm{al}^{8}$ reported that QSM has higher sensitivity and specificity than GRE phase imaging for differentiating calcifications and hemorrhages; however, in their study, the location and orientation of GRE phase images were not precisely defined. Examples of heterogeneous GRE phase images leading to the misinterpretation of the susceptibility of lesions in their study were correctly interpreted using our method. In addition to the border phase pattern, we could identify the susceptibility of lesions from the phase shift in the peripheral rim of the lesion in the axial central phase image, which revealed the opposite phase change to the susceptibility of the lesion. ${ }^{12}$ However, in our clinical experience, phase changes in the peripheral rim are weaker and less easy to interpret than the dipole itself, particularly in large lesions that have phase aliasing in the axial central phase images. They could also be affected by the local susceptibility change of the surrounding tissue, such as nearby veins.

The only lesion that was misinterpreted as a CMB based on its border phase pattern was a choroid plexus calcification at the foramen of Luschka. Calcifications could reveal paramagnetic susceptibility with extra depositions of paramagnetic substances, such as hemosiderin and ferrous or other metal components. This is a common finding in calcifications within the deep nucleus, particularly in the globus pallidum; however, this is rarely reported in calcifications outside the deep nucleus. ${ }^{8,9}$

Our study has several limitations. First, our method focused on only the phase patterns of GRE dark spots that are small $(<10 \mathrm{~mm}$ in diameter) and round or oval. The phase patterns may become heterogeneous in large lesions because of increased phase aliasing from high susceptibility effects and high-pass filtering. In addition, the dipole pattern caused by the susceptibility effect can differ in linear, tubular, and irregular lesions. However, the phase shift in the border section is less affected by the size and shape of the lesion. We observed that border phase patterns could be used to correctly identify the susceptibility of large and irregular lesions; however, additional studies should be conducted to confirm this finding. Second, the susceptibility of lesions with mixed hemorrhagic and calcified components cannot be precisely evaluated from the phase pattern because the phase image can only reveal the susceptibility of the dominant component, and this problem cannot be completely overcome through QSM. Third, if a calcification is accompanied by the deposition of paramagnetic substances, such as those in the deep nucleus or our false-negative case, this feature may be interpreted as a $\mathrm{CMB}$ from the phase pattern. However, this is also a limitation of QSM. In this situation, CT is still needed for the final diagnosis. Finally, it is important to know MR imaging vendors' phase presenting system (right-handed or left-handed system) to avoid making a wrong interpretation.

\section{CONCLUSIONS}

Our study revealed that border phase patterns in axial phase images can be a quick and robust reference for differentiating calcifications and CMBs in clinical MR imaging, and this technique had the same accuracy as QSM. Coronal reformation of the phase image can provide a favorable representation of the physical properties of magnetic dipoles caused by the susceptibility effect of target lesions.

\section{ACKNOWLEDGMENTS}

The authors thank Dr. Chien-Yuan Lin of GE Healthcare, Taiwan for his instruction on the physics of magnetic dipoles and QSM techniques.

\section{REFERENCES}

1. Greenberg S, Vernooij M, Cordonnier C, et al; Microbleed Study Group. Cerebral microbleeds: a field guide to their detection and interpretation. Lancet Neurol 2009;8:165-74 CrossRef Medline

2. Yates PA, Villemagne VL, Ellis KA, et al. Cerebral microbleeds: a review of clinical, genetic, and neuroimaging associations. Front Neurol 2013;4:205 CrossRef Medline

3. Haller S, Vernooij MW, Kuijer JPA, et al. Cerebral microbleeds: imaging and clinical significance. Radiology 2018;287:11-28 CrossRef Medline

4. Charidimou A, Shakeshaft C, Werring DJ. Cerebral microbleeds on magnetic resonance imaging and anticoagulant-associated intracerebral hemorrhage risk. Front Neurol 2012;3:133 CrossRef Medline

5. Kato $H$, Izumiyama $M$, Izumiyama $K$, et al. Silent cerebral microbleeds on T2*-weighted MRI: correlation with stroke subtype, stroke recurrence, and leukoaraiosis. Stroke 2002;33:1536-40 CrossRef

6. Mittal S, Wu Z, Neelavalli J, et al. Susceptibility-weighted imaging: technical aspects and clinical applications, Part 2. AJNR Am J Neuroradiol 2009;30:232-52 CrossRef Medline

7. Gupta RK, Rao SB, Jain R, et al. Differentiation of calcification from chronic hemorrhage with corrected gradient echo phase imaging. $J$ Comput Assist Tomogr 2001;25:698-704 CrossRef Medline

8. Chen W, Zhu W, Kovanlikaya I, et al. Intracranial calcifications and hemorrhages: characterization with quantitative susceptibility mapping. Radiology 2014;270:496-505 CrossRef Medline

9. Yamada N, Imakita S, Sakuma T, et al. Intracranial calcification on gradient-echo phase image: Depiction of diamagnetic susceptibility. Radiology 1996;198:171-78 CrossRef Medline

10. Wu Z, Mittal S, Kish K, et al. Identification of calcification with MRI using susceptibility-weighted imaging: a case study. J Magn Reson Imaging 2009;29:177-82 CrossRef Medline

11. Kaaouana T, de Rochefort L, Samaille T, et al. 2D harmonic filtering of MR phase images in multicenter clinical setting: toward a magnetic signature of cerebral microbleeds. Neuroimage 2015;104:287300 CrossRef Medline

12. Schweser F, Deistung A, Lehr BW, et al. Differentiation between diamagnetic and paramagnetic cerebral lesions based on magnetic susceptibility mapping. Med Phys 2010;37:5165-78 CrossRef Medline

13. Li W, Wu B, Liu C. Quantitative susceptibility mapping of human brain reflects spatial variation in tissue composition. Neuroimage 2011;55:1645-56 CrossRef Medline

14. de Rochefort L, Liu T, Kressler B, et al. Quantitative susceptibility map reconstruction from MR phase data using Bayesian regularization: validation and application to brain imaging. Magn Reson Med 2010;63:194-206 CrossRef Medline

15. Liu J, Liu T, de Rochefort L, et al. Morphology enabled dipole inversion for quantitative susceptibility mapping using structural consistency between the magnitude image and the susceptibility map. Neuroimage 2012;59:2560-68 CrossRef Medline 
16. Liu T, Wisnieff $\mathrm{C}$, Lou M, et al. Nonlinear formulation of the magnetic field to source relationship for robust quantitative susceptibility mapping. Magn Reson Med 2013;69:467-76 CrossRef Medline

17. Liu T, Liu J, de Rochefort L, et al. Morphology enabled dipole inversion (MEDI) from a single-angle acquisition: comparison with cosmos in human brain imaging. Magn Reson Med 2011;66:777-83 CrossRef Medline

18. Wang Y, Yu Y, Li D, et al. Artery and vein separation using susceptibility-dependent phase in contrast-enhanced MRA. J Magn Reson Imaging 2000;12:661-70 CrossRef Medline
19. Schenck JF. The role of magnetic susceptibility in magnetic resonance imaging: MRI magnetic compatibility of the first and second kinds. Med Phys 1996;23:815-50 CrossRef Medline

20. Axel L, Morton D. Correction of phase wrapping in magnetic resonance imaging. Med Phys 1989;16:284-87 CrossRef Medline

21. Conturo TE, Smith GD. Signal-to-noise in phase angle reconstruction: dynamic range extension using phase reference off-sets. Magn Reson Med 1990;15:420-37 CrossRef Medline 